\title{
Caracterización de cárcavas en las regiones VIII y IX de Chile
}

\author{
Gully characterization in the VIII ${ }^{\text {th }}$ and IX $^{\text {th }}$ Regions, Chile
}

C.D.O.: 116.2

ANDRES IROUME, JORGE GAYOSO, MARTA HERNANDEZ

Instituto de Manejo Forestal, Universidad Austral de Chile, Casilla 567, Valdivia, Chile.

\section{SUMMARY}

The gullies existing within an area of 66 thousand hectares were studied to characterize the affected area, location of affected terrains, level of activity, form, size (depth and surface), location in the slope, aspect, advancement rate, kind of affected resources and possible origin. The studied area is distributed in the provinces of Bio-Bio, Arauco and Malleco, in the VIII ${ }^{\text {th }}$ and IX ${ }^{\text {th }}$ Regions, Chile, concentrated mainly in the Nahuelbuta range of mountains, between the cities of Nacimiento and Angol.

The total area occupied with gullies is almost 450 hectares. Because gullies occurred associated with other forms of erosion, more than 6.000 hectares, which corresponds to $8 \%$ of the studied area, are estimated to be affected by some level of erosion.

Gullies are mainly located in terrains having soils derived from granitic materials. Although these terrains are now covered by plantation forests, $96 \%$ of the gullies are still active.

The mean depth and surface of gullies were 9.8 meters and 0.42 hectares, respectively.

The characteristics of the studied gullies show that $62 \%$ originated from agricultural practices before the forest development of the area. But, $26 \%$ was generated from unstable cut slopes and badly designed drainage systems of recently built forest roads.

The level of activity, size, location in the slope and advancement rate suggest the need to carry out especific gully control measures. Plantations have not controled the erosive process, which can be accelerated with harvesting and until the establishment of a new rotation.

\section{RESUMEN}

Las cárcavas existentes en un área de estudio de 66 mil hectáreas fueron estudiadas con el propósito de caracterizarlas en cuanto al área que ocupan, ubicación de los terrenos afectados, grado de actividad, forma, tamaño (profundidad y superficie), localización en la ladera, orientación, velocidad de avance, tipo de recurso afectado y posible origen. La superficie total estudiada se distribuye en las provincias de Bío-Bío, Arauco y Malleco, en las Regiones VIII y IX de Chile, concentrándose principalmente en la Cordillera de Nahuelbuta, entre Nacimiento y Angol.

La superficie ocupada por cárcavas alcanza cerca de 450 hectáreas. Puesto que las cárcavas corresponden a estados avanzados de fenómenos erosivos, se estima que al menos 6.000 hectáreas, es decir el $8 \%$ del área de estudio, estarían afectadas por algún nivel de erosión.

La mayor parte de las cárcavas se ubican en terrenos con suelos derivados de materiales graníticos. El $96 \%$ de las cárcavas están aún activas, a pesar de que los terrenos están actualmente cubiertos por bosques de plantaciones. La profundidad media y superficie promedio de las cárcavas individuales son 9.8 metros y 0.42 hectáreas, respectivamente.

Las características de las cárcavas muestran que un $62 \%$ se originaron por prácticas agrícolas anteriores al desarrollo forestal del área. Sin embargo, un $26 \%$ se ha generado de taludes de corte inestables y sistemas deficientes de drenajes en caminos forestales construidos más recientemente.

El grado de actividad, tamaño, localización en la ladera y velocidad de avance sugieren la necesidad de implementar medidas específicas de control de cárcavas. Las plantaciones no han detenido el proceso erosivo, el que puede acelerarse luego de la cosecha de los bosques, y hasta que una nueva rotación se establezca. 


\section{INTRODUCCION}

En 1958 se estimaba que 19 millones de hectáreas estaban erosionadas en Chile (Elizalde, 1958). Estudios más recientes indican que la erosión afecta a entre $34^{1}$ y 36 millones de hectáreas, es decir el $45 \%$ de la superficie total del país (Peralta, 1978). De acuerdo con esto, entre 1958 y 1991 la superficie erosionada ha aumentado en el país a una tasa cercana a las $450 \mathrm{mil}$ hectáreas por año. De esta cifra, la tasa de erosión irreversible sería de unas 50 mil hectáreas al año (CONAF, 1989). Las causas de esta degradación son en gran parte atribuibles a la actividad económica y no a procesos geológicos naturales.

Extensas áreas de la Cordillera de la Costa, que incluyen las provincias de Concepción, Bío-Bío, Malleco y Arauco, fueron convertidas en verdaderos graneros para abastecer de trigo los mercados generados desde mediados del siglo XIX y hasta fines de la década de 1920, tanto en el país como en el extranjero. El efecto combinado del monocultivo, de las técnicas de cultivo que araban las laderas en sentido de la máxima pendiente dejándolas descubiertas durante el período de mayores lluvias, y finalmente del método de inundación utilizado como sistemas para riego, generó un paulatino agotamiento de los suelos (Elizalde, 1958).

La intensidad de cultivo, sumado a las características topográficas y clima del sector, generaron graves procesos erosivos que comenzaron a hacerse evidentes ya desde fines del siglo pasado. En muchos casos, las zonas que se utilizaron para los cultivos de cereales y viñas de secano eran lomajes con pendientes relativamente fuertes a escarpadas, que correspondían a terrenos de aptitud ganadera o netamente forestal (IREN-CORFO, 1970).

Parte de la importancia de los procesos erosivos debe atribuirse también a las características de erodabilidad de los suelos, especialmente los de origen granítico que cubren grandes extensiones del área mencionada. Estos suelos presentan un bajo contenido de material coloidal o cohesivo, especialmente material orgánico que promuevan la agregación y cohesión, lo que los hace muy inestables (IREN-CORFO, 1970).

Otros factores que influyeron en la degradación del área fueron: el régimen de tenencia de la tie-

1 CIPMA, Cuarto Encuentro sobre el Medio Ambiente, Valdivia, 6-8 de mayo 1992. Grupo de Trabajo Manejo de Cuencas. rra, que por estar muy subdividida muchas veces dificultaba el acceso a asistencia técnica y económica que permitiera introducir mejoras productivas y métodos de conservación de suelos; y la ubicación de los caminos rurales y senderos en las partes altas de los cerros y con deficientes sistemas de drenaje (IREN-CORFO, 1970).

En la zona central de la Cordillera de la Costa la erosión ha sido tan intensa que la destrucción alcanza niveles de regiones administrativas. La magnitud y extensión del fenómeno erosivo en el área han sido descritas por IREN (1965), IRENCORFO (1970, 1979), Peña y Carrasco (1992), Peña et al. (1992), Sandoval et al. (1995) y Peña (1995).

La economía agraria, basada principalmente en el cultivo de cereales, pero también en las viñas de secano, prevaleció en el área de la Cordillera de la Costa de Chile Central hasta comienzos de la década de los 70. Desde esa fecha, gran parte de estos terrenos erosionados comenzaron a ser forestados con plantaciones de especies introducidas, lo que en cierta medida ha ayudado a detener los procesos de degradación. Sólo en la VIII Región, hacia 1993 existían sobre 700 mil hectáreas de plantaciones de Pino radiata y eucalipto (INFOR, 1994).

Sin embargo, en estos terrenos actualmente bajo un régimen de uso forestal es posible identificar sectores donde el establecimiento y el manejo de las plantaciones es difícil. Estos sectores están asociados a zonas severamente erosionadas, en las que la presencia y avance de cárcavas activas están afectando a las plantaciones establecidas y provocando daños de importancia a los caminos, entre otros.

Aun cuando gran parte de los terrenos están cubiertos por plantaciones forestales, se siguen produciendo grandes volúmenes de escurrimientos superficiales y las cárcavas siguen activas. Esto se debe sin duda a un desequilibrio hidrológico por el estado general de compactación de los suelos y por la menor eficiencia de la cobertura vegetal actual respecto a la original, en cuanto al balance hídrico y protección del suelo (Alvarez, 1988; Campos, 1990). También, a los efectos que las actividades silvícolas, principalmente las faenas de cosecha, imponen a la estabilidad de los suelos (Iroumé et al., 1989; Gayoso, Iroumé, 1991).

El uso agrícola como al que fueron sometidos estos terrenos genera mayores niveles de compactación en los suelos respecto a la condi- 
ción original (Ellies et al., 1993; Ellies, 1995). Aun cuando la mayor compactación de estos suelos puede tender a recuperarse parcialmente en el tiempo (Perry, 1964), la condición de los suelos favorece el escurrimiento superficial y al mismo tiempo dificulta el establecimiento de la vegetación (Hatchell et al., 1970; Gayoso et al., 1991).

Un nivel cobertura como la que ofrecen actualmente las plantaciones del área de estudio tiende en general a disminuir las pérdidas de suelo (Endlicher, 1982; Peña, 1978; Iroumé et al., 1989). Sin embargo, esta cubierta de árboles no detiene necesariamente el avance de cárcavas (Heede, 1976). La agresividad de estos sistemas puede ser superior a la presencia de la vegetación, puesto que está relacionada con las condiciones de pendiente de las paredes, el flujo del escurrimiento superficial sobre ellas y el desagüe de las aguas infiltradas y de los depósitos subterráneos a través de las cárcavas (Lassen et al., 1967; Andion, 1984).

Incluso bosques de plantaciones establecidos en terrenos degradados pueden aumentar el riesgo de movimiento en grandes masas del suelo, en lugar de disminuirlo (Hawley, Dymond, 1988). Esto es especialmente cierto en el caso de plantaciones coetáneas establecidas muy cerca de los bordes de cárcavas o de taludes inestables, ya que al alcanzar los árboles en forma simultánea un determinado peso, pueden generarse o reactivarse deslizamientos por las caídas de los mismos.

Por otra parte, la actividad forestal requiere una mayor densidad de caminos que la necesaria en áreas agrícolas (Losa, 1979; Wüthrich, 1992). La construcción de caminos altera las líneas naturales del escurrimiento del agua, generando una mayor erosión de las zonas comprometidas (Kochenderfer, Aubertin, 1975; Ottens, 1975; Rothwell, 1978; Rice et al., 1979; Swift, 1984a, 1984b; Fahey, Coker, 1989; Grayson et al., 1993).

Finalmente, las intervenciones forestales sobre estos bosques de plantaciones afectan severamente el ciclo hídrico, favoreciendo los escurrimientos superficiales y aumentando las tasas de erosión de los suelos (Rothwell, 1978; Rice et al., 1979; Sidle, 1980; Gayoso, Iroumé, 1991; Ursic, 1991; Grayson et al., 1993; Oyarzún, 1993). Los mayores efectos se producen durante la cosecha, que incluye la corta de los árboles, la construcción de huellas de madereo y caminos para extraer los árboles, el uso de maquinaria pesada para el madereo, y eventualmente el uso del fuego u otro tipo de disposición de desechos.
Empresas forestales establecidas en el área de estudio están considerando la posible recuperación de los terrenos afectados por cárcavas, para eventualmente incorporarlos al patrimonio productivo.

El objetivo de este estudio es el de localizar y cuantificar la superficie afectada por cárcavas, dentro de un patrimonio de 66 mil hectáreas. Además, caracterizar estas formas de erosión respecto al grado de actividad, ubicación, tipo de recursos afectados y posibles causas de origen. También, estimar la velocidad de avance de las cárcavas.

Los resultados de este estudio ayudarán a decidir respecto a la necesidad de implementar medidas específicas de control de cárcavas y recuperación de los terrenos degradados.

\section{DESCRIPCION DEL AREA DE ESTUDIO}

El área de estudio se enmarca entre los $37^{\circ} 15^{\prime}$ y $\operatorname{los} 38^{\circ} 15^{\prime}$ latitud sur y $73^{\circ} 15^{\prime}$ y $71^{\circ} 45^{\prime}$ longitud oeste, de la VIII y IX región, provincias de BíoBío, Arauco y Malleco, Ocupa principalmente la vertiente oriental de la Cordillera de Nahuelbuta. Al interior de estos límites se ubican las casi 66 mil hectáreas, agrupadas en 144 predios forestales, que fueron estudiadas en detalle para caracterizar las cárcavas.

El clima del área está clasificado como templado mediterráneo con estaciones secas y lluviosas semejantes. Los antecedentes disponibles muestran una precipitación media anual de $1.400 \mathrm{~mm}$, la cual se distribuye en un $26 \%$ en otoño, $55 \%$ en invierno, $14 \%$ en primavera y un $5 \%$ en verano (Hernández, 1992). Las lluvias se concentran entre los meses de mayo a agosto y su principal característica es su elevada erosividad (Brito, Peña, 1980).

La temperatura media es de $13.8^{\circ} \mathrm{C}$ y los vientos dominantes durante otoño e invierno son los norte y noroeste, y en primavera-verano los vientos sur (Hernández, 1992).

En esta área de la Cordillera de la Costa se presentan suelos con materiales granitoideos como es el caso de los suelos de las series San Esteban y Cauquenes, y suelos derivados de rocas y conglomerados volcánicos como lo son las series Huaqui y Collipulli. Una extensa descripción de los suelos en el área de estudio aparece en Hernández (1992).

El área presenta una topografía ondulada a quebrada y a veces disectada, con alturas de 100 hasta 1.000 m s.n.m, con pendientes promedio de aproxi- 
madamente un 35\% (Hernández, 1992). El área posee una red hidrográfica caracterizada por la presencia de los ríos Bío-Bío y Vergara, de numerosos esteros de caudal generalmente perennes que descargan en ellos, y de una serie de quebradas con régimen estacional, que determinan fuertemente las características topográficas del área.

La vegetación natural presente en el área es del tipo matorral nativo, con densidades variables (Hernández, 1992). Generalmente son renuevos de tocón, con numerosos pies por planta, y la calidad no las hace maderables, destinándolas principalmente a leña o carbón. La vegetación remanente se encuentra principalmente como protección de quebradas y cursos de agua. Las especies dominantes son: Nofhofagus obliqua (roble) Peumus bolclus (boldo), Litraea caustica (litre), Aristotelia chilensis (maqui), Rosa moschata (mosqueta) y otras especies como radal, mardonio, álamo y peumo, entre otras. En esta zona se presenta el tipo forestal esclerófilo degradado.

\section{MATERIAL Y METODO}

El estudio se realizó en 144 predios forestales pertenecientes a Forestal Mininco S.A. ${ }^{2}$ Se analizó la información proveniente de los planos rodalizados con curvas de nivel a escala 1:10.000, fotografías aéreas verticales color, escala promedio 1:10.000 desde 1981 en adelante, y fotografías blanco y negro de 1965 y otros vuelos hechos por la SAF, a escalas 1:50.000 y 1:60.000, además de inventarios y estudios pertinentes para elaborar planes de manejo y construcción de caminos disponibles para cada predio estudiado.

Para localizar los predios afectados por cárcavas se usaron las fotografías aéreas disponibles. Dentro de los predios se localizaron los sistemas de cárcavas, determinándose el rodal o ubicación topográfica general (Hernández, 1992). Se clasificó como cárcava al sector por el cual se canalizan las aguas, el que generalmente presenta el área inmediata fuertemente lavada. Es decir, en el cálculo de la superficie de cárcavas no se cuantificó ni el área de drenaje ni el cono de depósito de las mismas.

2 Forestal Río Vergara S.A. (FORVESA) al momento de realizar el estudio.
Junto con localizar los sistemas se delimitó el área de influencia del canal o cuerpo de la cárcava, sin considerar el área tributaria ni cono de depositación. Se traspasó la información de las fotografías a los planos escala 1:10.000, mediante Sketch-master, y la superficie de cada sistema se calculó según Hernández (1992).

Los sistemas de cárcavas fueron estudiados para caracterizarlos según IREN (1965), López (1968). Heede (1976) y Peralta (1977), identificando: estados de desarrollo, grado de actividad, ubicación en la ladera, recursos que están siendo afectados, posible origen, y características propias de estas formas avanzadas de erosión como profundidad, superficie, longitud de la pendiente, exposición, cobertura vegetal, tipo de erosión, tipo de cárcava y forma de la cárcava.

La caracterización se realizó para una muestra de los sistemas de cárcavas individualizados, y la información de cada cárcava muestreada fue registrada en un formulario especial (Hernández, 1992).

El nivel de actividad se determinó para cada una de las tres partes que constituyen una cárcava. Estas son: la cabeza, que generalmente presenta taludes casi verticales y donde se concentra el escurrimiento de las aguas provenientes del área de influencia de la cárcava; la garganta, por donde las aguas pueden escurrir en un sector más o menos encauzado; y el canal de desagüe, sector de menor pendiente donde tienden a depositarse los materiales de suelo arrastrados por las aguas. Se realizaron análisis estadísticos para obtener correlaciones entre las variables obtenidas.

La estimación de la velocidad de avance de las cárcavas se realizó en los sistemas erosivos de uno de los predios muestreados. El predio elegido fue San Antonio, ubicado en el área de Nacimiento, que presenta la casi totalidad de los sistemas activos, de superficies superiores a la media muestral, de gran longitud y ubicadas en fuertes pendientes (Hernández, 1992).

La estimación misma se realizó analizando una secuencia de fotografías aéreas del predio, de los años 1961 (escala 1:50.000). 1978 (escala 1:30.000), y 1985, 1987 y 1990 (escala 1:10.000). La determinación del avance consideró la zona de desprendimiento de suelo, traspasándose la información de cada una de la secuencia de fotos a través de un Sketch-master a un plano. En este plano se registraron las superficies de los sistemas en cada período de tiempo, determinándose de esta manera la diferencia de extensión de las cárcavas, 


\section{CUADRO 1}

Superficie y predios afectados según áreas

administrativas de la empresa.

Affected surface and areas.

\begin{tabular}{|c|c|c|c|c|c|c|c|}
\hline \multirow{3}{*}{ Areas } & \multicolumn{3}{|c|}{ Superficie } & \multicolumn{3}{|c|}{ Predios } & \multirow[t]{3}{*}{ Sene suelo* } \\
\hline & \multirow{2}{*}{$\begin{array}{c}\text { Estudiada } \\
\text { (ha) }\end{array}$} & \multicolumn{2}{|c|}{ Afectada } & \multicolumn{3}{|c|}{ Total afectados } & \\
\hline & & (ha) & $(\%)$ & $\left(\mathrm{N}^{\circ}\right)$ & $\left(\mathrm{N}^{\circ}\right)$ & $(\%)$ & \\
\hline Nacimiento & 14.115 & 60.54 & 40.01 & 20 & 13 & 65.0 & ET-CQ \\
\hline Nahuelbuta & 11.675 & 50.10 & 33.12 & 40 & 8 & 20.0 & ET \\
\hline Maintenrehue & 8.163 & 30.93 & 20.45 & 32 & 13 & 40.6 & ET \\
\hline Angol & 12.658 & 6.82 & 4.50 & 33 & 3 & 9.0 & ET \\
\hline Mulchén & 7.854 & 2.90 & 1.92 & 16 & 2 & 12.5 & HQ-CL \\
\hline Collipulli & 11.146 & 0.00 & 0.00 & 5 & 0 & 0.0 & CL \\
\hline Total & 65.647 & 151.29 & 100.00 & 146 & 39 & 100.0 & \\
\hline
\end{tabular}

*Series de suelos: $\quad$ ET $=$ San Esteban $($ granítico $)$

$\mathrm{CQ}=$ Cauquenes $($ granítico)

HQ = Huaqui (volcánico)

$\mathrm{CL}=$ Collipulli (volcánico)

lo que representa una estimación del avance lineal de las cárcavas.

\section{PRESENTACION Y DISCUSION DE RESUL- TADOS}

La superficie total afectada por el canal de las cárcavas alcanza a poco más de 151 hectáreas. En el cuadro 1 se resume la superficie y número de predios del área de estudio.

Las 151 hectáreas mencionadas no consideran el cono de depositación del material ni el área de drenaje de la cárcava. Según Heede (1960, 1966), al considerar tanto el canal como el área de drenaje y cono de depósito la superficie total de influencia de la cárcava, la superficie directamente afectada por estos sistemas erosivos alcanzaría a cerca de 450 hectáreas. Esto es menos del $1 \%$ de la superficie estudiada.

Aun cuando este porcentaje es sensiblemente menor a los que presentan IREN-CORFO (1979), Peña y Carrasco (1992), Peña et al. (1992), Sandoval et al. (1995) y Peña (1995) para la zona, las diferencias podrían explicarse por el método de cuantificación. En este estudio se midió la superficie atribuible directamente a las cárcavas.

Las cárcavas corresponden a un estado avanzado del fenómeno erosivo de un área determinada, y en el área de estudio se identificó además la presencia de erosión de manto, canalículos y canales o regueros (Hernández, 1992). Estas formas de erosión generalmente anteceden a las cárcavas (López, 1968; Heede, 1976; Peralta, 1977), por lo que en definitiva la superficie severamente afectada por erosión debe ser mucho mayor.

Según las cifras de CIPMA (CIPMA, Cuarto Encuentro sobre el Medio Ambiente, Valdivia, 68 de mayo 1992. Grupo de Trabajo Manejo de Cuencas) para Chile, la superficie total con algún nivel de erosión sería superior a 14 veces la afectada por cárcavas. Manteniendo esta proporción, se podría estimar que cerca de 6.000 hectáreas, que representan poco más del $8.3 \%$ de la superficie total estudiada, estarían afectadas por algún nivel de erosión, situación que debe estar influyendo negativamente en la productividad de estos sitios.

Con respecto a la localización, poco más del $98 \%$ de la superficie afectada por cárcavas se concentra en las áreas ${ }^{3}$ de Nacimiento, Nahuelbuta, Maitenrehue y Angol, que en total agrupan el $71 \%$ de la superficie estudiada (cuadro 1). El $2 \%$ res-

3 El estudio se realizó en terrenos de propiedad de Forestal Mininco S.A. Las áreas mencionadas corresponden a las divisiones administrativas que al momento del estudio tenía esta empresa. 
tante se localiza en el área de Mulchén, donde se concentra el $12 \%$ de la superficie estudiada. En el área de Collipulli, que agrupa el $17 \%$ de la superficie estudiada, no se identificaron cárcavas.

Los predios agrupados en las áreas de $\mathrm{Naci-}$ miento, Nahuelbuta, Maitenrehue y Angol están localizados sobre suelos de las series San Esteban y Cauquenes, que son ricos en materiales granitoideos. En las otras dos áreas los predios estudiados están sobre suelos derivados de rocas y conglomerados volcánicos (series Huaqui y Collipulli en Mulchén, y serie Collipulli en el área del mismo nombre).

Esta situación confirma a Peralta (1976) y Schlatter y Gerding (1985), entre otros, en relación con la mayor susceptibilidad a la erosión por agua de los suelos derivados de materiales graníticos, en los que se hace típica la formación de cárcavas.

La caracterización de las cárcavas se realizó a partir de una muestra altamente representativa de estas formas de erosión del área de estudio. Puesto que la gran mayoría de las cárcavas se presentan sobre suelos de origen granítico, el estudio en detalle se concentró en áreas erosionadas ubicadas sobre este tipo de suelos.

La muestra abarcó casi 73 hectáreas erosionadas (el $48.17 \%$ del total afectado) y 158 de las 394 cárcavas identificadas $(40.1 \%$ del total de cárcavas individuales). El detalle de la muestra se presenta en el cuadro 2.

Los principales resultados de la caracterización son los siguientes:

Localización en la ladera y exposición de las cárcavas: un $70 \%$ de las cárcavas muestreadas estaban localizadas a media ladera. Esto explica el nivel de actividad de las cárcavas, situación que es analizada más adelante en este mismo capítulo.

Un $17 \%$ está ubicado en la cima de las laderas. Según Peralta (1977), esta situación permitiría una estabilización natural de los sistemas.

Finalmente, un $13 \%$ está al pie de las laderas. Aun cuando estas cárcavas puedan presentar un nivel de base estable, las características de pendientes en las cabezas permiten pronosticar una erosión remontante.

E1 $73 \%$ de las cárcavas de la muestra están en laderas con exposiciones norte y un $90 \%$ en laderas convexas. Terrenos con estas características de exposición y forma de ladera son más favorables para los cultivos por la luz y drenaje. Sin embargo, los suelos con exposición norte están más ex-

\section{CUADRO 2}

Predios y superficie de la muestra, superficie y número de los sistemas individuales.

Location and surface of sample, Area and number of individual systems.

\begin{tabular}{|c|c|c|c|c|}
\hline \multirow[t]{2}{*}{ Area } & \multirow{2}{*}{$\begin{array}{c}\text { Predio } \\
\text { Superficie } \\
\text { (ha) }\end{array}$} & \multicolumn{2}{|c|}{$\begin{array}{r}\text { Cárcava } \\
\text { Superficie }\end{array}$} & \multirow{2}{*}{$\begin{array}{c}\text { Número } \\
\left(\mathrm{N}^{\circ}\right)\end{array}$} \\
\hline & & (ha) & $(\%)$ & \\
\hline \multicolumn{5}{|l|}{ NACIMIENTO } \\
\hline Liñeco & 648.4 & 23.00 & 3.54 & 69 \\
\hline Meñir & 2.999 .7 & 10.25 & 0.34 & 10 \\
\hline Sn. Antonio & 1.313 .0 & 4.42 & 0.34 & 17 \\
\hline Sn. Pedro & & & & \\
\hline de Carrizal & 447.4 & 4.51 & 1.01 & 15 \\
\hline Sta. Adriana & 1.754 .0 & 4.30 & 0.25 & 10 \\
\hline Total Area & 7.162 .5 & 46.48 & 0.65 & 121 \\
\hline \multicolumn{5}{|l|}{ NAHUELBUTA } \\
\hline Nicaicura & 62.5 & 7.61 & 12.18 & 5 \\
\hline Sn. José Grande & e 201.9 & 11.66 & 5.78 & 18 \\
\hline Total Area & 264.4 & 19.27 & 7.29 & 23 \\
\hline \multicolumn{5}{|l|}{ MAITENREHUE } \\
\hline Los Pantanos & 161.4 & 2.50 & 1.55 & 3 \\
\hline La Bodega & 86.5 & 1.40 & 1.62 & 2 \\
\hline Total Area & 247.9 & 3.90 & 1.57 & 5 \\
\hline \multicolumn{5}{|l|}{ ANGOL } \\
\hline Casablanca & 344.9 & 1.40 & 0.41 & 4 \\
\hline Verdun & 536.1 & 1.92 & 0.36 & 5 \\
\hline Total Area & 881.0 & 3.32 & 0.38 & 9 \\
\hline Total muestra & 8.555 .8 & 72.87 & 0.85 & 158 \\
\hline
\end{tabular}

puestos a erosividad de los agentes climáticos, son más erosionables y presentan generalmente una cubierta vegetal natural menos densa y menos variada (IREN-CORFO, 1970).

No se encontró ninguna correlación entre pendiente de la ladera y ocurrencia de cárcavas. Se observaron cárcavas tanto en sectores prácticamente planos como en sectores escarpados. Sin duda que la presencia de cárcavas en terrenos de pendientes moderadas refleja la degradación de los suelos debido al mal uso agrícola a que fueron sometidos.

Forma y tamaño de las cárcavas: de la muestra estudiada, un $47 \%$ de las cárcavas tenía forma lineal. Peralta (1977) asocia esta forma al laboreo en el sentido de la máxima pendiente y a la inadecuada canalización del agua. 


\section{CUADRO 3}

Magnitudes medias de las cárcavas y longitud de pendiente en los predios muestreados. Average magnitude of gullies and length of slopes in the sampled area.

\begin{tabular}{|c|c|c|c|c|c|c|c|}
\hline \multirow{3}{*}{$\begin{array}{l}\text { AREA } \\
\text { Predio }\end{array}$} & \multicolumn{5}{|c|}{ CÁRCAVAS } & \multicolumn{2}{|c|}{$\begin{array}{l}\text { LONGITUD DE LA PENDIENTE } \\
\qquad(\mathrm{m})\end{array}$} \\
\hline & \multirow[t]{2}{*}{ Número } & \multicolumn{2}{|c|}{ Profundidad (m) } & \multicolumn{2}{|c|}{ Superfi cié (ha) } & \multirow[b]{2}{*}{ Media } & \multirow[b]{2}{*}{ D.V.S. } \\
\hline & & Media & D.V.S. & Media & D.V.S. & & \\
\hline \multicolumn{8}{|l|}{ NACIMIENTO } \\
\hline Liñeco & 69 & 8.5 & 6.4 & 0.53 & 0.65 & 322.9 & 159.7 \\
\hline Meñir & 10 & 16.4 & 9.6 & 0.56 & 0.48 & 375 & 2.33 .7 \\
\hline Sn. Antonio & 17 & 9.4 & 8.7 & 0.26 & 0.24 & 298.2 & 187.1 \\
\hline Sn. Pedro & & & & & & & \\
\hline de Carrizal & 15 & 6.9 & 5.2 & 0.24 & 0.21 & 242 & 120.4 \\
\hline Sta. Adriana & 10 & 14.1 & 6.4 & 0.41 & 0.29 & 295 & 122.3 \\
\hline \multicolumn{8}{|l|}{ NAHUELBUTA } \\
\hline Nicaicura & 5 & 8.6 & 4.1 & 1.38 & 1.11 & 498 & 279.0 \\
\hline Sn. José Grande & 18 & 7.8 & 4.3 & 0.40 & 0.35 & 271.1 & 87.8 \\
\hline \multicolumn{8}{|l|}{ MAITENREHUE } \\
\hline Los Pantanos & 3 & 15.8 & 2.8 & 0.83 & 0.45 & 500 & 122.5 \\
\hline La Bodega & 2 & 27.5 & 7.5 & 0.70 & 0.15 & 300 & 0.0 \\
\hline \multicolumn{8}{|l|}{ ANGOL } \\
\hline Casablanca & 4 & 11.4 & 5.9 & 0.35 & 0.21 & 400 & 127.5 \\
\hline Verdúm & 5 & 12.8 & 7.3 & 0.38 & 0.31 & 1.420 & 153.6 \\
\hline Total & 158 & 9.8 & 7.4 & 0.42 & 0.56 & 3.535 & 256.0 \\
\hline
\end{tabular}

E1 $30 \%$ de las cárcavas tenía forma compuesta, en la que se combinan diversas formas de cárcavas. Según Peralta (1977), esta forma se asocia a estados avanzados de desarrollo de las cárcavas, las que por el nivel de actividad se unen, dando origen a un gran sistema de forma compleja, de mayor superficie activa, más agresivo y difícil de controlar. Puesto que estas cárcavas no fueron en ningún momento controladas, actualmente se presentan profundas, activas y ocupando frecuentemente el largo de la ladera.

E1 $23 \%$ restante tiene forma bulbosa, en la cual es posible identificar varias pequeñas cabezas. Este tipo de cárcavas también se asocia a estados avanzados de desarrollo.

De las cárcavas estudiadas, el $87 \%$ eran cárcavas continuas. E1 $13 \%$ restante correspondía a cárcavas discontinuas que formaban un sistema en cadena de cárcavas de relativo menor tamaño.

Con respecto a su tamaño, según la clasificación dada por Peralta (1977) corresponden a cárcavas grandes, con profundidades promedio de 9.8 metros (cuadro 3). La superficie media de las cárcavas individuales fue de 0.42 hectáreas.

Se observó una tendencia, estadísticamente no significativa, entre la superficie de las cárcavas, ubicación en la ladera, longitud de la pendiente y exposición. Las cárcavas de mayor superficie (mayores a la media) se ubican en longitudes de pendiente menores a la media, principalmente en la cima de la ladera y en exposiciones norte. En cambio las cárcavas de superficie menor a la media se encuentran preferentemente en la basé de la ladera. Igualmente las cárcavas de mayor superficie presentan profundidades superiores a la media muestral de $9.8 \mathrm{~m}$ (cuadro 3). Coincidiendo con 10 que indica Peralta (1977), los sistemas de mayor superficie presentan en su mayoría la forma compuesta, debido al grado de desarrollo alcanzado.

Desarrollo de las cárcavas: la mayor parte de las cárcavas se encuentra en los dos primeros estados de desarrollo, descritos por Peralta (1977). 
El primero corresponde al inicio de un proceso de formación de cárcavas, el cual afecta los horizontes superficiales. El segundo estado, que es el más activo y difícil de manejar, se caracteriza por la profundización y avance que afecta el subsuelo. En sectores donde existe en profundidad material blando, la socavación es acelerada y se producen desmoronamientos sucesivos que forman prácticamente paredes laterales.

Algunas cárcavas se encuentra en el tercer estado de desarrollo (Hernández, 1992). Este se caracteriza porque la tierra que ha caído de los desmoronamientos se acumula al pie de los taludes y las pendientes de las paredes se van suavizando, lo que ayuda a la colonización de pastos, hierbas y arbustos.

Origen de las cárcavas: analizando el estado de desarrollo, forma y localización en las laderas, se puede concluir que el $62 \%$ de las cárcavas estudiadas tiene un origen asociado a prácticas agrícolas anteriores al desarrollo forestal de área.

Un $26 \%$ de las cárcavas debe su origen a la construcción de caminos forestales recientes. La mayor frecuencia de estas cárcavas se observó en caminos temporales, en los cuales por el menor uso se invierten menores recursos en la construcción de la calzada, estabilización de taludes y evacuación del agua. La concentración de los escurrimientos y la falta de protección en los desagües ha generado normalmente cárcavas lineales cuyas cabezas se forman justamente en la descarga de los sistemas de evacuación.

E1 $12 \%$ restante de las cárcavas se ha originado en cauces naturales de fuerte pendiente. Estas cárcavas estarían también asociadas a la actividad forestal, y podrían deberse a la deforestación de las quebradas. El uso del fuego como elemento de preparación de los terrenos para plantación, que en áreas de fuertes pendientes es muy difícil de controlar, ha sido la causa de la eliminación de la cubierta vegetal en muchas quebradas. Estos cursos naturales quedan desprotegidos, y pueden activarse movimientos de masas que dan origen a cárcavas.

Recurso afectado: en cuanto al recurso afectado, el $60 \%$ de las cárcavas está comprometiendo a plantaciones forestales. Numerosos árboles caen al interior de las cárcavas, las que en su avance disminuyen la superficie útil para plantar. Sin embargo, tal vez el mayor problema es que estos sistemas erosivos dividen el terreno, dificultando las faenas de manejo, construcción de vías de acceso y especialmente las de cosecha.
E1 $40 \%$ restante de las cárcavas está afectando a caminos. Los mayores problemas están asociados a incisiones en los taludes de corte y terraplén por la concentración de los caudales de desagüe y por el depósito de los materiales erosionados que obstruyen los sistemas de drenaje. En algunos casos los caminos fueron construidos en laderas que presentaban cárcavas, y al no haberse considerado medidas de control, éstos están muy deteriorados o amenazados por el nivel de actividad y avance de estos sistemas. En otros casos, caminos temporales están siendo afectados por las cárcavas generadas por los deficitarios o inadecuados sistemas de evacuación de las aguas con los que fueron construidos.

Velocidad de avance de las cárcavas: la estimación del avance de las cárcavas se realizó por medio de una secuencia de fotografías aéreas del predio San Antonio.

Las fotografías de los años 1960 y 1970 son de carácter general, y por la escala $(1: 50.000,60.000)$ se hizo difícil la determinación de una distancia de avance de los sistemas. En las fotografías escala promedio 1:10.000 más recientes, pertenecientes a la empresa propietaria de los terrenos, fue más fácil determinar la variación de avance.

Considerando el período 1985-1990, se estimó que las cárcavas han avanzado de 10 a $50 \mathrm{~cm}$ al año. Aun cuando este rango parece amplio, es muy similar al determinado por Alvarez (1988) en la misma área de estudio. Peña (1995), en terrenos localizados en la cuenca del río Itata, registró 18 $\mathrm{cm}$ de avance en la cabecera de cárcavas en un lapso de 6 meses. Puesto que las precipitaciones en los períodos de estío son prácticamente nulas, el grado de avance puede considerarse representativo a nivel anual.

Las cárcavas estudiadas presentan zonas de mayor avance relativo. En éstas, durante los períodos de lluvia, se producen desmoronamientos en los bordes en bloques de 1 a $2 \mathrm{~m}^{2}$ aproximadamente, de mayor longitud que ancho. Esto ocurre con mayor frecuencia en la cabecera de la cárcava, pero también en la garganta cuando las pendientes son excesivas.

Actividad de las cárcavas: en relación al nivel de actividad, poco más del $96 \%$ de las cárcavas muestreadas están activas. Durante el estudio, sólo 6 de las 158 cárcavas muestreadas aparecían estables. En el resto se notan desprendimientos de suelo y arrastre, observándose material suelto en las paredes y bordes y depósitos en el nivel base 
(Hernández, 1992). Hay escasa vegetación al interior de las cárcavas, las que muestran un aspecto lavado. Generalmente los taludes tienen fuertes pendientes que dificultan el poblamiento vegetal.

Entre las partes que constituyen una cárcava, el mayor nivel de actividad se registró en la cabeza y garganta, que en el 92 y $85 \%$, respectivamente, de las cárcavas estaban activas. El canal de desagüe resultó activo en el $62 \%$ de las cárcavas estudiadas.

El relativo mayor grado de estabilidad en el canal de desagüe en relación a las otras partes de las cárcavas lo explica Peralta (1976) en base a que esta zona presenta una menor pendiente por la acumulación de los aportes de material, que incluye gran parte del suelo fértil arrastrado, que promueve el establecimiento de la vegetación. Esta vegetación actúa como barrera al arrastre y ayuda al aterramiento natural y estabilización de la cárcava.

Llama la atención el alto porcentaje de cárcavas activas. A pesar de que en su mayoría éstas se originaron por prácticas agrícolas ocurridas con anterioridad al desarrollo forestal, podría esperarse una mayor estabilización de estos sistemas erosivos por la cobertura vegetal que ofrecen actualmente los bosques de plantaciones del área.

Sin embargo, el nivel de actividad confirma a Heede (1976), respecto a que la vegetación no detiene necesariamente el avance de las cárcavas. Alvarez (1988) y Campos (1990) sostienen además que la actual cobertura posee una menor eficiencia que la cubierta de bosque original en cuanto al balance hídrico y protección del suelo.

Por otra parte, los procesos de cosecha de estos bosques de plantaciones reactivan los procesos erosivos al quedar los suelos bruscamente sin ninguna cobertura vegetal. Según Oyarzún (1993), las mayores tasas de erosión se producen en cosecha a tala rasa y donde los residuos se queman como una forma de preparar el sitio para una nueva plantación. Iroumé et al. (1989) señalan que durante el año que sigue a la cosecha a tala rasa se producen más del $45 \%$ de las pérdidas de suelo por erosión que ocurren durante una rotación. La protección efectiva de la nueva plantación comienza al cuarto o quinto año después de establecida, período durante el cual se producen grandes pérdidas de suelo y fertilidad (Heede, 1976).

Las mayores tasas de erosión se producen en áreas cuyos bosques son cosechados a tala rasa, y donde los residuos se queman como una forma de preparar el sitio para una nueva plantación. Una forma de reducir las pérdidas de suelo en el perío- do entre la cosecha y el establecimiento de la nueva plantación es manteniendo los residuos de la explotación sobre la superficie de los terrenos intervenidos (Peña, 1978; Oyarzún, 1993).

Los bosques establecidos no han modificado las condiciones específicas que originaron a las cárcavas. Puesto que un alto porcentaje de éstas están activas y ubicadas a media ladera, y presentan un nivel de base y de cabecera inestables, es de esperar que continúen remontando la ladera para tender a estabilizarse sólo al llegar a la cima.

Además, zanjas de infiltración construidas como medida de control pero ubicadas demasiado cerca de la cabeza de algunas cárcavas han favorecido aún más el nivel de actividad. Esto, ya que el agua que infiltra aflora al pie de las paredes provocando inestabilidad y desprendimiento de bloques de tierra.

El estado de desarrollo de las cárcavas y los bienes e infraestructura que están siendo afectados refuerzan la necesidad de implementar medidas específicas para el control de estos sistemas erosivos, adicionales a la reforestación de las áreas que las rodean y a la construcción esporádica de zanjas de infiltración para reducir el escurrimiento en las laderas.

El control debe combinar medidas bioingenieriles o biomecánicas para estabilizar las cárcavas mismas. Entre éstas se pueden mencionar: establecer vegetación al interior y en las paredes de las cárcavas, estabilizar los taludes, construir diques para atrapar sedimentos y consolidar el fondo de las cárcavas, y disminuir artificialmente las pendientes de las paredes (Heede, 1960; 1966; 1976; Agpada et al. 1976; Peralta, 1977; Grey, Leiser, 1982).

Una serie de medidas adicionales debe considerarse en relación a la construcción de caminos forestales. Esto, ya que un porcentaje importante de las cárcavas está asociado a sistemas de evacuación de aguas de algunos caminos. Algunas medidas que se pueden recomendar son: planificar la construcción de modo de evitar sectores naturalmente inestables; proteger los taludes de corte y terraplén contra la erosión y los deslizamientos; y diseñar adecuados sistemas de evacuación de las aguas (Schiecht1, 1986; Gayoso, 1994).

\section{CONCLUSIONES}

La superficie total afectada directamente por cárcavas es menos del $1 \%$ del área estudiada. Sin 
embargo, ya que las cárcavas indican niveles avanzados de degradación de los suelos, se estima que unas 6.000 hectáreas, el $8 \%$ de la superficie estudiada, estarían afectas a niveles de erosión, cuya magnitud está afectando la productividad de los terrenos involucrados.

En el área estudiada, la mayoría de los sistemas de cárcavas identificados se localizan sobre suelos de origen granítico, mientras que estas formas de erosión son casi inexistentes en suelos volcánicos. Estos resultados confirman la alta susceptibilidad a la erosión por agua de los suelos derivados de materiales graníticos, en los que se hace típica la formación de cárcavas.

La orientación, forma y pendiente de las laderas y el tipo mayoritario de cárcavas presente en el área de estudio permiten identificar como el principal origen de estas formas erosivas al uso agrícola a que los terrenos estuvieron sometidos. Sin embargo, un porcentaje preocupante de éstas debe su origen a la construcción más reciente de caminos forestales, principalmente a sistemas deficientes de evacuación de aguas.

A pesar de que los terrenos del área de estudio están en la actualidad reforestados, poco más del $96 \%$ de las cárcavas están activas, y su velocidad de avance se estima entre 10 a $50 \mathrm{~cm}$ al año. Esto confirma a diversos autores en el sentido de que la vegetación no detiene necesariamente el avance de las cárcavas. Esto se debe, por una parte, a que la actual cobertura posee una menor eficiencia que la cubierta de bosques original en cuanto al balance hídrico y protección del suelo, y por otra, a que la agresividad de estos sistemas puede ser superior a la presencia de la vegetación, ya que está relacionada con las condiciones de pendiente de las paredes, el flujo del escurrimiento superficial sobre ellas y el desagüe de las aguas infiltradas y de los depósitos subterráneos a través de las cárcavas.

El nivel de actividad y el estado de desarrollo de las cárcavas y los bienes e infraestructura que están siendo afectados refuerzan la necesidad de implementar medidas específicas para el control de estos sistemas erosivos. Estas medidas deben incluir intervenciones en las cárcavas mismas. También, planificar la red de caminos de modo de evitar sectores inestables y diseñar sistemas adecuados de evacuación de las aguas.

\section{AGRADECIMIENTOS}

Los autores agradecen a Forestal Mininco S.A. (Forestal Río Vergara S.A. al momento de la investigación) el apoyo y financiamiento para realizar este estudio.

\section{BIBLIOGRAFIA}

AGPADA, A., D. ENDANGAN, S. FESTIN, J. GUMAYAGAY, TH. HOENNINGER, G. SEEBER. K. UNKEL, H.J. WEIDELT. 1976. Manual of reforestation and erosion control for the Philippines. German Agency for Technical Cooperation (GTZ), $492 \mathrm{pp}$.

ALVAREZ, F. 1988. Evaluación de la erosión producida por actividades forestales productivas. Ministerio de Agricultura. Corporación Nacional Forestal, Programa de Manejo de Cuencas VIII Región. Chile, 34 pp.

ANDION, A. 1984. Estudio para el control y estabilización de la cárcava de Quitirisi, Cantón de Mora. Programa de Cooperación del Gobierno de España. Santiago de Puriscal. España. 33 pp.

BRITO. O., J.L. PEÑA. 1980. "Determinación del factor "R" de la ecuación universal de predicción de pérdidas de erosión hídrica en la provincia de Nuble". Agricultura Técnica Chile 40:152-156.

CAMPOS. D. 1990. Efectos ecológicos de los nuevos esquemas de manejo del Pino radiata, Bosque Arauco S.A. CORMA: 41-45.

CONAF. 1989. Memoria programa Manejo de Cuencas. 19801989. Santiago, s/p.

ELIZALDE, R. 1958. La sobrevivencia de Chile. Ministerio de Agricultura. Santiago. Chile, $57 \mathrm{pp}$.

ELLIES, A. 1995. "Modificaciones en las propiedades físicas de suelos Palehumults y Hapludands debido al manejo". Actas Simposio IUFRO para Cono Sur Sudamericano Manejo Nutritivo de Plantaciones Forestales. Valdivia, Chile, 25-30 abril 1995, 255-270.

ELLIES, A., R. MACDONALD, C. RAMIREZ. 1993. "Variación de la estructura de un suelo forestal sujeto a distintos manejos", Boletín $\mathrm{N}^{\circ} 10$, VIII Simposio de la Sociedad Chilena de la Ciencia del Suelo, Valdivia, Chile, 8-10 abril 1993, 118-134.

ENDLICHER, W. 1982. "Instalación de parcelas de experimentación para evaluar la erosión del suelo en la Cordillera de la Costa de Chile Central", Actas III Congreso Geológico Chileno. Concepción, Chile, 11 pp.

FAHEY, B.D., R.J. COKER. 1989. "Forest Road Erosion in the Granite Terrain of Southwest Nelson, New Zealand". Journal of Hydrology (N.Z.) 28:123-141.

GAYOSO, J. 1994. Situación de impacto ambiental de la red de caminos del fundo Nonguén. Informe de Convenio, Universidad Austral de Chile, Valdivia (circulación restringida), $62 \mathrm{pp}$.

GAYOSO, J., A. IROUME. 1991. "Compaction and soil disturbances from logging in Southern Chile". Ann. Sci. For. 48:63-71.

GAYOSO, J., A. IROUME, A. ELliES. 1991. "Degradación de suelos forestales asociada a operaciones de cosecha". En: Actas III Taller de Producción Forestal Fundación Chile. Concepción, 27-29 noviembre.

GRAYSON, R.B., S.R. HAYDON, M.D.A. JAYASURUYA, B.L. FINLAYSON. 1993. "Water quality in mountain ash forests, separating the impacts of roads from those of logging operations", Journal of Hydrology 150: 459-480. 
GRAY, D.H., A.T. LEBER. 1982. Biotechnical slope protection and erosion control. Van Nostrand Reinhold Company Inc., New York, 271 pp.

HATCHELL, G., C. RALSTON, R. FOIL. 1970. "Soil disturbance in logging". J. Forestry. 68: 772-775.

HAWLEY, J., J. DYMOND. 1988. "How much do tree reduce landsliding". Reprinted from J. of Soil and Water Conservation, 43.

HEEDE. B. 1960. A study of early-control structures in the Colorado front range. USDA Forest Service, Rocky Mountain Forest and Range Experiment Station, Station Paper $\mathrm{N}^{\circ} 55,42 \mathrm{pp}$

HEEDE, B. 1966. Design construction and cost of rock check dams. USDA Forest Service, Research Paper RM-20, 24 pp.

HEEDE, B. 1976. Gully development and control: the status of our knowledge. USDA Forest Service, Research Paper RM$169,42 \mathrm{pp}$

HERNANDEZ, M. 1992. Cuantificación, caracterización y jerarquización de cárcavas en predios forestales del área de Nacimiento. Tesis, Facultad de Ciencias Forestales, Universidad Austral de Chile, 71 pp.

INFOR. 1994. "Estadísticas Forestales 1993", Boletín Estadístico 38. Santiago, Chile, $80 \mathrm{pp}$.

IREN. 1965. Evaluación de la erosión: Cordillera de la Costa entre Valparaíso y Cautín. Santiago, Chile, 12 pp.

IREN-CORFO. 1970. Manejo destructivo de la tierra. Cordillera de la Costa. Provincia de Concepción. Santiago, Chile, $22 \mathrm{pp}$.

IREN-CORFO. 1979. Fragilidad de los ecosistemas naturales de Chile. Informe 40. Santiago, Chile, 43 pp.

IROUME. A., J. GAYOSO, L. INFANTE. 1989. "Erosión hídrica y alteración del sitio en cosecha a tala rasa", Rev. Ecol. Biol. Sol. 26:171-180.

KOCHENDERFER, J.M., G.M. AUBERTIN. 1975. "Effects of management practices on water quality and quantity: Fernow Experimental Forest, West Virginia", En: Proc Municipal Watershed Management Symp. USDA Forest Service Gen. Tech. Report NE-13. 14-24.

LASSEN, L., H. LULL, B. FRANK. 1967. Algunas relacione entre planta, suelo y agua en el manejo de cuencas. División de Influencias Forestales. Servicio Forestal. Centro regional de Ayuda Técnica. Agencia para el Desarrollo Internacional. Circular $\mathrm{N}^{\circ}$ 910. México, $67 \mathrm{pp}$

LOPEZ, F. 1968. Aspectos cualitativos y cuantitativos de la erosión hídrica $y$ del transporte $y$ depósito de material. Madrid, España, 187 pp.

LOSA, J. 1979. Caminos económicos. Ed. Mundi-Prensa, Madrid, España, 269 pp.

OTTENS, J. 1975. Environmental costs in logging road design and construction. Environmental Canada, Forestry Service, Report BC-X-108, 26 pp

OYARZUN, C. 1993. "Evaluación del modelo U.S.L.E. para predecir pérdidas de suelo en áreas forestadas de la cuenca del río Bío-Bío", Bosque 14: 45-54.

PEÑA, L. 1978. "Control de erosión mediante dos niveles de densidad de paja en un trumao de lomaje". Agricultura Técnica Chile 38: 49-53.

PEÑA, F. 1995. "Evaluación y zonificación de la erosión hídrica en el secano costero de la cuenca del río Itata, VIII Re- gión". VII Congreso Nacional de la Ciencia del Suelo. Temuco, Chile, 10-13 marzo 1995, p. 87.

PEÑA, L., P. CARRASCO. 1992. "Clasificación preliminar de erosión en los suelos de la cuenca del río Bío-Bío". Gestión de los Recursos Hídricos de la Cuenca del Río Bío-Bío y del Area Marina Costera Adyacente. Serie Propuestas de Ordenamiento, Volumen 4: Elementos cogniscitivos sobre el Recurso Suelo y Consideraciones Generales sobre el Ordenamiento Agroforestal. Concepción. Chile: 33-44 pp.

PEÑA, L., P. CARRASCO, M. FIGUEROA, C. OYARZUN, B. LO CASCIO. 1992. "Pérdidas por erosión hídrica en suelos agrícolas y forestales de la cuenca del río Bío-Bío". Gestión de los Recursos Hídricos de la Cuenca del río BioBio y del Area Marina Costera Adyacente, Serie Propuestas de Ordenamiento. Volumen 4: Elementos Cognoscitivos sobre el Recurso suelo y Consideraciones Generales sobre el Ordenamiento Agroforestal. Concepción, Chile: 47-60.

PERALTA, M. 1976. Uso. clasificación y conservación tie suelos. Chile, 337 pp.

PERALTA, M. 1977. Las cárcavas y su control. Universidad de Chile, Facultad de Ciencias Forestales, Manual $N^{\circ} 5.82$ pp.

PERALTA, P. 1978. Procesos y áreas de desertificarían en Chile Continental. Mapa Preliminar. Ciencias Forestales. Vol. $1 \mathrm{~N}^{\circ} 1$, Santiago.

PERRY, T. 1964. "Soil compaction and loblolly pine growth". Tree Planter's Notes. 67: 9

RICE. R.M.. F. TILlEY. P.A. DATZMAN. 1979. A watershed's response to logging and roads: South Fork Creek. California. 1967-1976. USDA Forest Service. Res. Paper PSW-146, 12 pp.

ROTHWELL, R.L. 1978. Watershed management guideline for logging and road construction in Alberta. Environmental Canada, Forestry Service, Inf. Rep. NOR-X-208, 43 pp.

SANDOVAL, M., L. PEÑA, P. CARRASCO. 1995. "Una visión preliminar de erosión en los suelos de la cuenca del río Bío-Bío". VII Congreso Nacional de la Ciencia del Suelo. Temuco, Chile, 10-13 marzo 1995, p. 43.

SCHLATTER, J., V. GERDING. 1985. "Deficiencia de boro en plantaciones de Pinus radiata D.Don en Chile. I: Distribución y origen", Bosque 6:24-31.

SCHIECHTL, H. 1986. Manual de estabilización de cuencas hidrográficas. Estabilización de laderas con tratamientos de suelo y la vegetación. Guía FAO Conservación 13/1. Roma, Italia, 65 pp.

SIDLE, R.C. 1980. Impacts of forest practices on surface erosion. Pacific Northwest Extension Publication PNW 195. $15 \mathrm{pp}$.

SWIFT, L.W. 1984a. "Gravel and grass surfacing reduces soil loss from mountain roads". Forest Sci. 30: 657-670.

SWIFT, L.W. JR. 1984b. "Soil losses from roadbeds and cut and fill slopes in the Southern Appalachian Mountains", Southern J. of Applied Forestry. 8: 209-215.

URSIC. S.J. 1991. "Hydrological effects of two methods of harvesting mature Southern pine", Wat. Resour. Bull. 27:303-315.

W ÜTHRICH. W. 1992. Die Feinerschliesssung von Waldbeständen. Berichte der Eidgenössischen Forschungsanstalt fur Wald, Schnee und Landschaft $\mathrm{N}^{\circ} 332,92 \mathrm{pp}$. 